\title{
Optimal coordinated beamforming with artificial noise for secure SWIPT in multi-cell networks
}

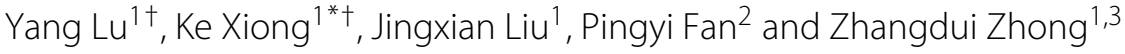

\begin{abstract}
This paper investigates the multi-cell coordinated beamforming (MCBF) design for secure simultaneous wireless information and power transfer (SWIPT) in both centralized and distributed manners. In each cell, one transmitter serves multiple information receivers (IRs) and energy receivers (ERs) with the non-linear energy harvesting (EH) model. Meanwhile, several eavesdroppers (Eves) intend to intercept the confidential information transmitted for IRs. To achieve a secure transmission, the artificial noise (AN) is embedded in the transmit signals of each transmitter. The proposed design is formulated into a power-minimization problem to guarantee the IRs' information and ERs' energy requirements while avoiding the information being intercepted by Eves. Since the problem is non-convex and not easy to solve, a solution method based on semi-definition relaxation (SDR) is proposed and the global optimum is proved to be guaranteed with full channel state information (CSI). We further present a distributed AN-aided MCBF for the system by using alternating direction method of multipliers (ADMM), with which each transmitter is able to calculate its own beamforming vectors and AN covariance matrix based on its local CSI. Simulation results show that our proposed distributed design converges to the global optimum obtained by the centralized one. It is also shown that by employing AN, the total required power of the system is reduced and the effect of AN on the system performance decreases with increment of transmit antennas. Compared with traditional linear EH model, optimizing the system under the non-linear EH one avoids false output power at the ERs and saves power at the transmitter.
\end{abstract}

Keywords: Green communications, SWIPT, Beamforming, Artificial noise, ADMM

\section{Introduction}

\subsection{Background}

Recently, simultaneous wireless information and power transfer (SWIPT) has attracted increasing interests, where the same radio frequency (RF) signals are used for transmitting both energy and information. Thus, SWIPT is expected to prolong the lifetime of low-power energyconstrained networks, such as wireless sensor networks (WSNs) and Internet of Things (IoT) [1-4]. Compared with traditional wireless communication system, SWIPT is equipped with the energy harvesting (EH) module, where the RF-EH circuits enable energy receiver (ERs) to scavenge wireless energy by converting the received RF

\footnotetext{
*Correspondence: kxiong@bjtu.edu.cn

${ }^{\dagger}$ Equal contributors

'School of Computer and Information Technology, Beijing Jiaotong University, Haidian District, 100044 Beijing, People's Republic of China

Full list of author information is available at the end of the article
}

signals into output direct current (DC) power. As for the RF-EH circuit, one of the most important performance measurement indexes is the RF-to-DC conversion efficiency factor, which is the ratio of the input power in the $\mathrm{RF}$ signals and the output DC power. In most existing works, the RF-to-DC conversion efficiency is regarded as a constant taking value in $(0,1]$, referring to the linear $E H$ model. However, according to very recent works, [5-7], the RF-to-DC conversion efficiency turns out to depend on the level of the input power, which means RF-to-DC conversion efficiency varies based on the input power and cannot be regarded as a constant anymore, referring to the non-linear EH model. Therefore, to avoid the system performance loss, the transmit design for SWIPT should be redesigned based on the non-linear $\mathrm{EH}$ model.

Meanwhile, green communications and high spectral efficiency (SE) are two important demands for future 5G networks. To achieve green communications, one 
way is designing energy-efficient systems to achieve high energy efficiency (EE) [8], and the other way is designing energy-saving systems to consume as less power as possible to meet system requirements [9]. To achieve high SE, many advanced communication technologies were proposed, such as cooperative relaying [10, 11], coordinated beamforming [12-14], and network coding [15]. Among them, multi-cell coordinated beamforming (MCBF) $[12,14]$ is regarded as one of the most promising technologies to achieve low-power consumption and high $\mathrm{SE}$, as well as guaranteeing quality of service (QoS) for receivers in multi-cell multi-user systems, since it is capable of exploiting the spatial degree of freedom (DoF) to migrate the inter-cell and intra-cell interference. Compared with the single-cell beamforming design, MCBF is more challenging since inter-cell interference cannot be neglected. Moreover, it is relatively easy for a single-cell transmitter to acquire channel state information (CSI) of all receivers, but for MCBF, a control center is required to gather all the intra-cell CSI and the inter-cell CSI for all transmitters, and the exchange of CSI between the control center and transmitters brings heavy burden and overhead to the system. As the future wireless systems prefer a flat Internet Protocol (IP) architecture [16] where all transmitters are directly connected with the core network rather than the control center, distributed MCBF is required to be developed where all transmitters work at their local CSI.

Due to the broadcast nature, another important issue in multi-cell multi-user systems is to avoid the confidential information leakage from the information receivers (IRs) to the eavesdropper (Eves). To achieve information security, physical-layer secure transmission has drawn increasing attention [17-19]. The main idea is to embed the artificial noise (AN) into the transmit signal to confuse Eves so that only the IRs can decode the confidential information correctly.

\subsection{Motivation and contribution}

To inherit the advantages of MCBF and SWIPT as well as achieving secure transmission, in this paper, we investigate AN-aided MCBF designs for multi-cell multi-user SWIPT systems in both centralized and distributed manners. The contributions of our work is summarized as follows:

- To realize green communication, we formulate an optimization problem to minimize the total required power of the whole system while satisfying the following system requirements: (1) minimal information rate requirement at each IR, (2) minimal $\mathrm{EH}$ requirement at each ER, and (3) avoiding any IR to be intercepted by any Eve.

- With full CSI assumption, we optimize the system and solve the problem in a centralized manner. In this case, since the considered problem is non-convex and cannot be solved directly, we solve it by a proposed solution method based on semi-definition relaxation (SDR) and the global optimal solution is proved to be always achieved.

- With local CSI assumption, we also optimize the system and solve the problem in a distributed manner. In this case, each transmitter is able to calculate its own beamforming vectors and AN covariance matrix based on its local CSI. The proposed distributed design is derived based on alternating direction method of multipliers (ADMM).

- Numerous simulation results are provided to demonstrate our analysis, which show that our proposed distributed design converges to the global optimum obtained by the centralized one. We also observe that by employing AN, the total required power of the system is reduced and the effect of AN on the system performance decreases with increment of transmit antennas. Compared with a traditional linear EH model, optimizing the system under the non-linear EH model avoids a false output power at the ERs and also saves power at the transmitter.

\subsection{Related work}

Although MCBF and secure SWIPT has been investigated in the literature [19-31], they were separately discussed in various systems and only few works studied MCBF and secure SWIPT in a single system. In order to highlight the novelty of this paper, it is worth emphasizing the following differences between our work and existing ones [19-31].

Firstly, this is different from some existing works, see, e.g., $[19,20]$, where the system secrecy rate was maximized. In this paper, we consider the power-minimization design to cater for the requirement of green communications. The authors in $[19,20]$ formulated the problems as optimization (feasibility) problems and gave some approximate solutions based on the Bisection method. While we obtain the global optimal solution to our considered system and prove that $\mathrm{AN}$ also helps to save transmit power. Moreover, the inter-cell interference was not taken into consideration in these works.

Secondly, the power-minimization design was also investigated in some works, see, e.g., [21-27], but they all focused on single-cell SWIPT scenarios. Besides, they all adopted the ideal linear EH model, which mismatches the practical system and may lead to false optimization results of a system configuration. To avoid the performance loss, this paper considers the non-linear EH model obtained by real data measurement [5-7].

Thirdly, although MCBF designs were investigated for various systems (see, e.g, [28-31]), most of them did not consider SWIPT and communication secrecy. For instance, the total required power was minimized under 
information rate constraints in $[28,29]$, the sum rate was maximized with zero-forcing beamforming in [30], and the energy efficiency was maximized with massive MIMO transition in [31]. As SWIPT plays a very important role in future wireless communication and secure transmission is an inevitable issue in broadcast SWIPT systems, it is of high importance to present suitable AN-aided MCBF designs for multi-cell SWIPT systems. Therefore, in this paper, a secure SWIPT in multi-cell systems is investigated.

Fourthly, the existing works only designed the centralized AN-aided MCBF for secure SWIPT system, while in our work, we also present an efficient distributed design, where each transmitter could generate its transmit beamforming using only a local CSI. In our work, multiple kinds of receivers, i.e., IRs, ERs and Eves, are considered, which is more general and more practical in the future system.

This paper is organized as follows. Section 2 gives the system model description and the problem statement. Section 3 gives the optimal centralized non-robust design. The distributed design is given in Section 4. The simulation results are provided in Section 5. Finally, Section 6 concludes the paper.

Notations: Boldface lowercase and uppercase letters denote vectors and matrices, respectively. The set of all $\mathrm{n}$-by-m complex matrixes is denoted by $\mathbb{C}^{n \times m}$. For a complex number $a,|a|$ denotes the modulus. For a vector a, $\left\|\mathbf{w}_{n}\right\|_{2}$ denotes the Euclidean norm. The conjugate transpose, rank, trace and determinant of the matrix $\mathbf{A}$ are denoted as $\mathbf{A}^{H}$, rank (A), $\operatorname{Tr}(\mathbf{A})$, and $\operatorname{det}(\mathbf{A})$, respectively. $\mathbf{A} \succeq 0$ means $\mathbf{A}$ is a positive semidefinite (PSD) matrix. The symbol $\mathbf{I}$ denotes the identity matrix and $\mathbf{0}$ denotes a zero vector or matrix. The symbol $\mathbb{E}\{$.$\} represents the$ statistical expectation of the argument.

\section{System model and problem formulation}

\subsection{Networks model}

We consider a multi-cell multi-user downlink system with $N_{c}$ cells as shown in Fig. 1. Each cell consists of one $N_{\mathrm{t}}$-antenna transmitter, $K$ single-antenna IRs, $L$ singleantenna ERs, and $S$ single-antenna Eves. $N_{\mathrm{c}}$ transmitters transmit signals over a common frequency band, which means that the inter-cell and intra-cell interference coexist in the system. To prevent the information leakage, the energy-bearing spatially selective AN is embedded in the transmit signals of each transmitter.

For clarity, we use $k, l, s$, and $n$ to denote the $k$-th IR, the $l$-th ER, $s$-th Eve, and the $n$-th cell/transmitter, respectively, where $k \in \mathcal{K}, l \in \mathcal{L}, s \in \mathcal{S}$, and $n \in \mathcal{N}_{\mathrm{c}}$. $\mathcal{K} \triangleq[1,2, \ldots, K], \mathcal{L} \triangleq[1,2, \ldots, L], \mathcal{S} \triangleq[1,2, \ldots, S]$, and $\mathcal{N}_{\mathrm{c}} \triangleq\left[1,2, \ldots, N_{\mathrm{c}}\right]$ denote the sets of IRs, ERs, Eves, and cells/transmitters, respectively. Thus, the transmit signal from the $n$-th transmitter is

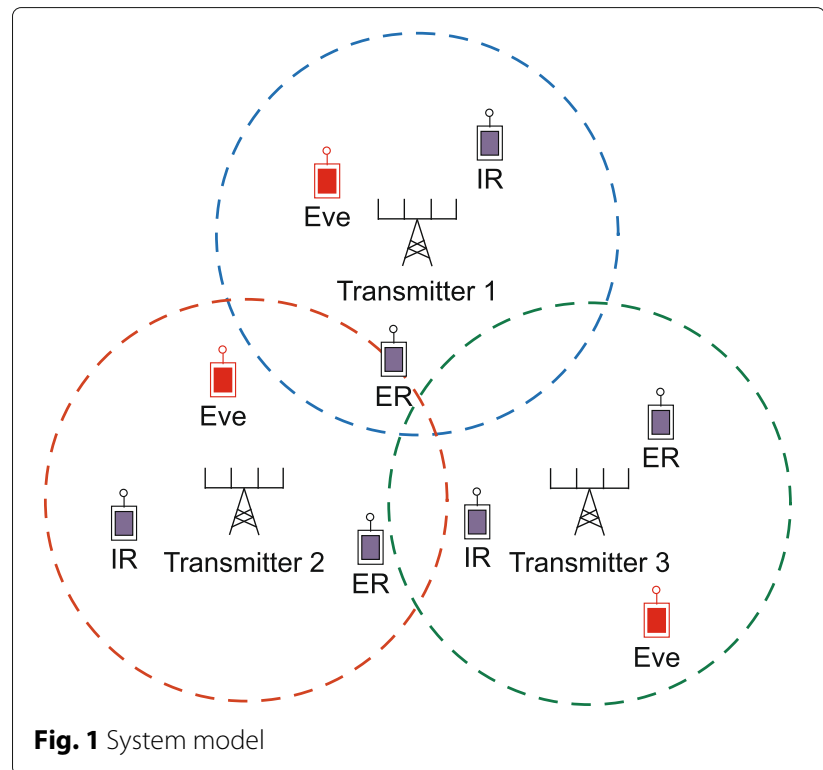

$$
\mathbf{x}_{n}(t)=\sum_{k=1}^{K} \mathbf{w}_{n k} \omega_{n k}(t)+\mathbf{z}_{n}(t) \in \mathbb{C}^{N_{\mathrm{t}} \times 1},
$$

where $\omega_{n k} \in \mathbb{C}$ with $\mathbb{E}\left\{\left|\omega_{n k}\right|^{2}\right\}=1$ is the data symbol for the $k$-th IR transmitted in the $n$-th cell. $\mathbf{w}_{n k} \in$ $\mathbb{C}^{N_{\mathrm{t}} \times 1}$ is the corresponding transmit beamforming vector. $\mathbf{z}_{n} \in \mathbb{C}^{N_{\mathrm{t}} \times 1}$ denotes the AN vector which follows Gaussian distribution, i.e., $\mathbf{z}_{n} \sim \mathcal{C N}\left(\mathbf{0}, \Sigma_{n}\right)$ and $\Sigma_{n} \succeq \mathbf{0}$. Thus, the total required power of the system can be given by $\sum_{n=1}^{N_{\mathrm{c}}}\left(\sum_{k=1}^{K}\left\|\mathbf{w}_{n k}\right\|_{2}^{2}+\operatorname{Tr}\left(\boldsymbol{\Sigma}_{n}\right)\right)$.

Then, the received signal at the $k$-th IR and the $l$-th ER in the $n$-th cell can be, respectively, given by

$$
\begin{aligned}
& \mathbf{y}_{n k}^{(\mathrm{IR})}(t)= \sum_{m=1}^{N_{\mathrm{c}}} \mathbf{h}_{m n k}^{H} \mathbf{x}_{m}(t)+n_{n k}(t) \\
&= \underbrace{\mathbf{h}_{n n k}^{H} \mathbf{w}_{n k} \omega_{n k}(t)}_{\text {desired signal }}+\underbrace{\sum_{i \neq k}^{K} \mathbf{h}_{n n k}^{H} \mathbf{w}_{n i} \omega_{n i}(t)}_{\text {intra-cell interference }} \\
&+\underbrace{\sum_{m \neq n}^{N_{\mathrm{c}}} \sum_{i=1}^{K} \mathbf{h}_{m n k}^{H} \mathbf{w}_{m i} \omega_{m i}(t)}_{\text {inter-cell interference }}+\underbrace{\sum_{m=1}^{N_{\mathrm{c}}} \mathbf{h}_{m n k}^{H} \mathbf{z}_{m}(t)}_{\mathrm{AN}}+n_{n k}(t), \\
& \mathbf{y}_{n l}^{(\mathrm{ER})}(t)=\sum_{m=1}^{N_{\mathrm{c}}} \mathbf{h}_{m n l}^{H} \mathbf{x}_{m}(t)+n_{n l}(t)
\end{aligned}
$$

and that at the $s$-th Eve in cell $n$ is

$$
\mathbf{y}_{n s}^{(\text {Eve })}(t)=\sum_{m=1}^{N_{\mathrm{c}}} \mathbf{g}_{m n s}^{H} \mathbf{x}_{m}(t)+v_{n s}(t),
$$

where $\mathbf{h}_{m n k}, \mathbf{h}_{m n l}$, and $\mathbf{g}_{m n s} \in \mathbb{C}^{N_{\mathrm{t}} \times 1}$ denote the channel vectors from the $m$-th transmitter to the $k$-th IR, the $l$-th $\mathrm{ER}$, and the $s$-th $\mathrm{Eve}^{1}$ in the $n$-th cell, respectively. $n_{n k}(t)$, $n_{n l}(t)$, and $v_{n s}(t)$ are the Gaussian noises with variance $\sigma_{n k}^{2}, \sigma_{n l}^{2}$, and $\sigma_{n s}^{2}$ at the $k$-th IR, the $l$-th ER, and the $s$-th Eve, respectively. Following (2) and (4), the received SINR at the $k$-th IR in cell $n$ and that at the $s$-th ER in cell $n$ are given by (5) and (6), respectively. 


$$
\begin{aligned}
& \operatorname{SINR}_{n k}\left(\left\{\mathbf{w}_{m 1}, \cdots \mathbf{w}_{m K}, \mathbf{\Sigma}_{m}\right\}_{m=1}^{N_{\mathrm{c}}}\right)=\frac{\left|\mathbf{h}_{n n k}^{H} \mathbf{w}_{n k}\right|^{2}}{\sum_{i \neq k}^{K}\left|\mathbf{h}_{n n k}^{H} \mathbf{w}_{n i}\right|^{2}+\sum_{m \neq n}^{N_{\mathrm{c}}} \sum_{i=1}^{K}\left|\mathbf{h}_{m n k}^{H} \mathbf{w}_{m i}\right|^{2}+\sum_{m=1}^{N_{\mathrm{c}}} \mathbf{h}_{m n k}^{H} \Sigma_{m} \mathbf{h}_{m n k}+\sigma_{n k}^{2}} \\
& \operatorname{SINR}_{n s}^{e}\left(\left\{\mathbf{w}_{m 1}, \cdots \mathbf{w}_{m K}, \boldsymbol{\Sigma}_{m}\right\}_{m=1}^{N_{\mathrm{c}}}\right)=\max _{k \in \mathcal{K}}\left(\frac{\left|\mathbf{g}_{n n s}^{H} \mathbf{w}_{n k}\right|^{2}}{\sum_{i \neq k}^{K}\left|\mathbf{g}_{n n k}^{H} \mathbf{w}_{n i}\right|^{2}+\sum_{m \neq n}^{N_{\mathrm{c}}} \sum_{i=1}^{K}\left|\mathbf{g}_{m n s}^{H} \mathbf{w}_{m i}\right|^{2}+\sum_{m=1}^{N_{c}} \mathbf{g}_{m n s}^{H} \boldsymbol{\Sigma}_{m} \mathbf{g}_{m n s}+\sigma_{n s}^{2}}\right)
\end{aligned}
$$

\subsection{Non-linear EH model}

Each ER converts the received RF signals into output DC power by its RF-EH circuits. At the $l$-th ER in the $n$-th cell, the input power of its RF-EH circuits from the received RF signals is

$$
\begin{aligned}
& P_{n l}^{(\mathrm{ER})}= \\
& \mathbf{h}_{m n k}^{H}\left(\sum_{n=1}^{N}\left(\sum_{k=1}^{K} \mathbf{w}_{n k} \mathbf{w}_{n k}^{H}+\boldsymbol{\Sigma}_{n}\right)\right) \mathbf{h}_{m n k} .
\end{aligned}
$$

In most existing works, the RF-to-DC conversion efficiency $\rho$ of the RF-EH circuits is regarded as a constant in the interval $(0,1]$, referring to the linear EH model, which indicates that the RF-to-DC conversion efficiency is independent of the input power level. However, in practice, the RF-EH circuits include various non-linearities, such as the diode or diode-connected transistor. As a result, the RFto-DC conversion efficiency depends on the input power level. To capture the dynamics of the RF-to-DC conversion efficiency for different input power levels, in this paper, the non-linear model is adopted [5-7]. The output DC power (harvested power) of the RF-EH circuits at the $s$-th ER is

$$
\Phi_{n l}\left(\left\{\mathbf{w}_{m 1}, \cdots \mathbf{w}_{m K}, \boldsymbol{\Sigma}_{m}\right\}_{m=1}^{N_{c}}\right)=\frac{\Psi_{n l}}{X_{n l}}-Y_{n l}
$$

with

$$
\Psi_{n l}=\frac{M_{n l}}{1+\exp \left(-a_{n l}\left(P_{n l}^{(\mathrm{ER})}-b_{n l}\right)\right)},
$$

where

$$
X_{n l}=\frac{\exp \left(a_{n l} b_{n l}\right)}{1+\exp \left(a_{n l} b_{n l}\right)}, Y_{n l}=\frac{M_{n l}}{\exp \left(a_{n l} b_{n l}\right)} .
$$

$\Psi_{n l}$ is a logistic function of $P_{n l}^{(\mathrm{ER})}, M_{n l}$ is a constant denoting the maximum output DC power, which indicates the saturation limitation of the RF-EH circuits. $a_{n l}$ and $b_{n l}$ are constants representing some properties of the $\mathrm{EH}$ system, e.g., the resistance, the capacitance and the circuit sensitivity. In general, $M_{n l}, a_{n l}$, and $b_{n l}$ depend on the choice of hardware components for assembling the $\mathrm{EH}$ system and can be estimated through a standard curve fitting algorithm. Figure 2 provides an example of the nonlinear EH model, where the maximum output DC power $M_{n l}$ is set as $20 \mathrm{~mW}$. One can observe that the output DC power increases with the increment of the input power at first, and then when it reaches the saturation region, the output DC power cannot surpass this saturation limitation, which is much different from the linear EH model, where the output DC power can always increases with the increment of the input power.

\subsection{Problem formulation}

Our goal is to minimize the total required power of the system by jointly optimizing the transmit beamforming vectors and covariance matrixes of the AN to meet the following two system requirements.

- To guarantee the information rate requirement of each IR, its received SINR should be larger than a predefined threshold $\gamma_{\mathrm{u}}$.

- To prevent the information interception of each Eve, its received SINR should be lower than a predefined threshold $\gamma_{\mathrm{e}}$.

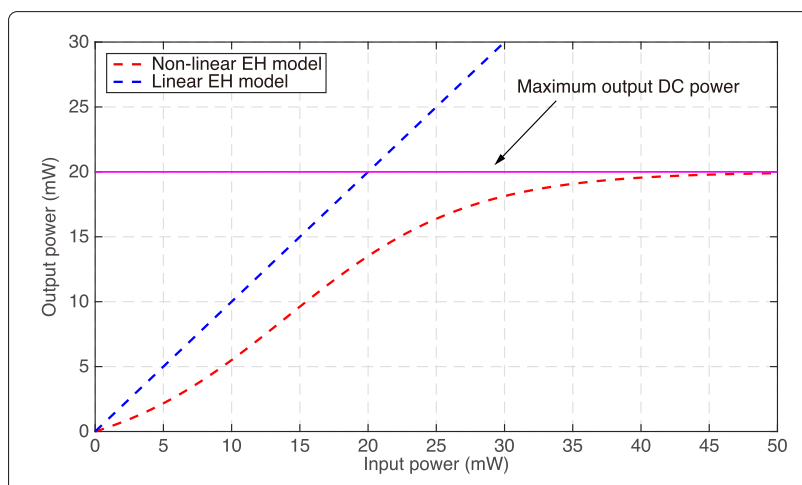

Fig. 2 Non-linear EH model where $M_{n l}$ is set as $20 \mathrm{~mW}$ 
- To guarantee the EH requirement of each ER, its output DC power should be larger than a predefined threshold $\theta$.

Then, our considered power-minimization AN-aided MCBF design is mathematically formulated as

$$
\begin{aligned}
& \min _{\left\{\mathbf{w}_{n k}\right\}\left\{\Sigma_{n}\right\}} \sum_{n=1}^{N_{\mathrm{c}}}\left(\sum_{k=1}^{K}\left\|\mathbf{w}_{n k}\right\|_{2}^{2}+\operatorname{Tr}\left(\Sigma_{n}\right)\right) \\
& \text { s.t. } \operatorname{SINR}_{n k}\left(\left\{\mathbf{w}_{m 1}, \cdots \mathbf{w}_{m K}, \boldsymbol{\Sigma}_{m}\right\}_{m=1}^{N_{c}}\right) \geq \gamma_{\mathrm{u}} \\
& \operatorname{SINR}_{n s}^{e}\left(\left\{\mathbf{w}_{m 1}, \cdots \mathbf{w}_{m K}, \boldsymbol{\Sigma}_{m}\right\}_{m=1}^{N_{c}}\right) \leq \gamma_{\mathrm{e}} \\
& \Phi_{n l}\left(\left\{\mathbf{w}_{m 1}, \cdots \mathbf{w}_{m K}, \boldsymbol{\Sigma}_{m}\right\}_{m=1}^{N_{\mathrm{c}}}\right) \geq \theta \\
& \boldsymbol{\Sigma}_{n} \geq \mathbf{0}, \forall n, m \in \mathcal{N}_{\mathbf{c}},
\end{aligned}
$$

With (9b) and (9c), the secrecy capacity between each IR and its serving transmitter is guaranteed bounded below $C_{\mathrm{sec}}=\log \left(1+\gamma_{\mathrm{u}}\right)-\log \left(1+\gamma_{\mathrm{u}}\right)$. Note that the value of $\gamma_{\mathrm{u}}$ and $\gamma_{\mathrm{e}}$ depends on the required QoS of IRs. A larger $\gamma_{\mathrm{u}}$ and a smaller $\gamma_{\mathrm{e}}$ indicate better system performance, but more power is required at transmitters.

Problem (9) is not convex due to the non-convex constraint (9b)-(9d), which cannot be solved directly. Therefore, in Section 3, we will solve it by using SDR methods ${ }^{2}$.

\section{Optimization in the power minimization design}

To solve problem (9), one solution method based on SDR is proposed, where SDR means letting $\mathbf{W}_{\mathbf{n k}}=\mathbf{w}_{\mathbf{n k}} \mathbf{w}_{\mathbf{n k}}{ }^{H}$ and removing the rank-one constraint.

The EH constraint (9d) under the non-linear EH model can be represented by

$$
\sum_{m=1}^{N} \mathbf{h}_{m n l}^{H}\left(\sum_{k=1}^{K} \mathbf{w}_{n k} \mathbf{w}_{n k}^{H}+\boldsymbol{\Sigma}_{n}\right) \mathbf{h}_{m n l} \geq \tau_{n l}
$$

where $\tau_{n l}$ indicates the required received power (input power of the RF-EH circuits) under the non-linear $\mathrm{EH}$ model, which can be given by

$$
\tau_{n l}=b_{n l}-\frac{\ln \left(\frac{M_{n l}}{\left(\theta+Y_{n l}\right) X_{n l}}-1\right)}{a_{n l}} .
$$

By employing SDR, the SDR form of (10) can be given by

$$
\operatorname{Tr}\left(\sum_{m=1}^{N} \mathbf{h}_{m n l}^{H}\left(\sum_{k=1}^{K} \mathbf{W}_{n k}+\boldsymbol{\Sigma}_{n}\right) \mathbf{h}_{m n l}\right) \geq \tau_{n l},
$$

Then, the SDR form of problem (9) can be given by

$$
\begin{array}{ll} 
& \min _{\left\{\mathbf{W}_{n k}, \Sigma_{n}\right\}} \sum_{n=1}^{N_{c}}\left(\sum_{k=1}^{K} \operatorname{Tr}\left(\mathbf{W}_{n k}\right)+\operatorname{Tr}\left(\Sigma_{n}\right)\right) \\
\text { s.t. } & \operatorname{Tr}\left(\mathbf{h}_{n n k} \mathbf{h}_{n n k}^{H} \mathbf{U}_{n k}\right) \\
\geq & \sum_{m \neq n}^{N_{c}} \operatorname{Tr}\left(\mathbf{h}_{m n k} \mathbf{h}_{m n k}^{H} \Theta_{m}\right)+\sigma_{n k}^{2}, \\
& \operatorname{Tr}\left(\mathbf{g}_{n n s} \mathbf{g}_{n n s}^{H} \Xi_{n k}\right) \\
\leq & \sum_{m \neq n}^{N_{c}} \operatorname{Tr}\left(\mathbf{g}_{m n s} \mathbf{g}_{m n s}^{H} \Theta_{m}\right)+\sigma_{n s}^{2} \\
& (11), \mathbf{W}_{n k} \succeq \mathbf{0}, \Sigma_{n} \succeq \mathbf{0}, \\
& \forall k \in \mathcal{K}, \forall l \in \mathcal{L}, \forall s \in \mathcal{S}, \forall n, m \in \mathcal{N}_{\mathrm{c}} .
\end{array}
$$

where

$$
\begin{aligned}
\mathbf{U}_{n k} & =\left(\frac{1}{\gamma_{u}} \mathbf{W}_{n k}-\sum_{i \neq k}^{k} \mathbf{W}_{n i}-\Sigma_{n}\right), \\
\Xi_{n k} & =\left(\frac{1}{\gamma_{e}} \mathbf{W}_{n k}-\sum_{i \neq k}^{k} \mathbf{W}_{n i}-\Sigma_{n}\right), \\
\Theta_{n} & =\left(\sum_{k=1}^{k} \mathbf{W}_{n k}+\Sigma_{n}\right) .
\end{aligned}
$$

By doing so, problem (9) is expressed into a convex problem, i.e., problem (12). By using some off-the-shelf solvers, e.g., SeduMi or CVX, the optimal solution, i.e., $\mathbf{W}_{n k}^{\star}$ and $\Sigma_{n}^{\star}$, to problem (12) can be obtained. Note that, our goal is to obtain the optimal $\mathbf{w}_{n k}^{\star}$ rather than $\mathbf{W}_{n k}^{\star}$. Therefore, once we get $\mathbf{W}_{n k}^{\star}$, we should recover $\mathbf{w}_{n k}^{\star}$ from $\mathbf{W}_{n k}^{\star}$. Generally, only when $\mathbf{W}_{n k}^{\star}$ is rank-one, problems (9) and (12) have the same optimal result and solution, which means that only in this case, $\mathbf{w}_{n k}^{\star}$ can be recovered by rank-one decomposition of $\mathbf{W}_{n k}^{\star}$ without loss.

Proposition 1 The rank-one solution to problem (12) always exists.

Proof The proof of Proposition 1 can be found in the Appendix of this paper.

Following Proposition 1, the optimal solution to problem (9) can be obtained by firstly solving problem (12) and then applying rank-one decomposition to the optimal solution of Problem (12).

Proposition 2 Compared with the MCBF without $A N$ (i.e., the non AN-aided MCBF), the AN-aided MCBF required less power under the same condition.

Proof The non AN-aided MCBF design can be formulated by setting the objective function of (9) as

$$
\min _{\left\{\mathbf{w}_{n k}\right\}} \sum_{n=1}^{N_{\mathrm{c}}} \sum_{k=1}^{K}\left\|\mathbf{w}_{n k}\right\|_{2}^{2}
$$

and eliminating $\left\{\Sigma_{m}\right\}_{m=1}^{N_{c}}$ in (9b), (9c), and (9d). It also can be solved by our proposed SDR-based solution method. 
One can see that the optimal solution to the non ANaided MCBF is a feasible solution to problem (9) by setting $\left\{\Sigma_{n}\right\}_{n=1}^{N_{\mathrm{c}}}=\mathbf{0}$. So the optimal result of problem (9) must be smaller than that of the non AN-aided MCBF, which means the $\mathrm{AN}$-aided $\mathrm{MCBF}$ required less power than the non AN-aided MCBF to satisfy the same system requirements.

Proposition 2 indicates that by introducing the AN to the $\mathrm{MCBF}$, the total required power of the system is reduced.

\section{Distributed design with ADMM}

Although we solve problem (9) efficiently in Section 3 by using SDR and obtain the global optimal solution, the proposed solution method is in a centralized manner, which means that to calculate the optimal beamforming vectors and AN covariance matrices, the CSI of all receivers in the system is required at each transmitter. Such a requirement yields heavy burden and overhead to the network, which blocks the deployment of the centralized design. Therefore, designing a distributed $\mathrm{AN}$-aided MCBF, where each transmitter only work with its local CSI, is of high importance. In this section, we shall apply ADMM to decentralize the proposed centralized AN-aided MCBF.

The main idea of the proposed distributed algorithm is to decompose the primal centralized problem into $N_{\mathrm{c}}+1$ subproblems, so that each transmitter can deal with its own beamforming vectors and AN covariance matrix based on local CSI at every iteration.

The first ingredient of the proposed distributed design is to find the penalty augmented problem of (12). For this, we define some slack variables as follows:

$$
\begin{aligned}
p_{n} & =\left(\sum_{k=1}^{K} \operatorname{Tr}\left(\mathbf{W}_{n k}\right)+\operatorname{Tr}\left(\boldsymbol{\Sigma}_{n}\right)\right), \\
t_{m n k} & =\operatorname{Tr}\left(\mathbf{h}_{m n k} \mathbf{h}_{m n k}^{H} p_{m}\right), T_{n k}=\sum_{m \neq n}^{N_{c}} t_{m n k}, \\
e_{m n s} & =\operatorname{Tr}\left(\mathbf{g}_{m n s} \mathbf{g}_{m n s}^{H} p_{m}\right), E_{n s}=\sum_{m \neq n}^{N_{c}} e_{m n s}, \\
q_{m n l} & =\operatorname{Tr}\left(\mathbf{h}_{m n l} \mathbf{h}_{m n l}^{H} p_{m}\right), Q_{n k}=\sum_{m \neq n}^{N_{c}} q_{m n k} .
\end{aligned}
$$

Then, problem (12) can be equally rewritten as

$$
\begin{gathered}
\left.\min \sum \sum_{n k}\right\}\left\{\Sigma_{n}\right\}\left\{p_{n}\right\}\left\{T_{n k}\right\}\left\{t_{m n k}\right\} \\
\left\{Q_{n l}\right\}\left\{q_{m n l}\right\}\left\{E_{n s}\right\}\left\{e_{m n s}\right\} \\
\text { s.t. } \\
\operatorname{Tr}\left(\mathbf{h}_{n n k} \mathbf{h}_{n n k}^{H} \mathbf{U}_{n k}\right) \geq T_{n k}+\sigma_{n k}^{2}, \\
\operatorname{Tr}\left(\mathbf{g}_{n n s} \mathbf{g}_{n n s}^{H} \mathbf{\Xi}_{n k}\right) \leq E_{n s}+\sigma_{n s}^{2}, \\
\operatorname{Tr}\left(\mathbf{h}_{n n l} \mathbf{h}_{n n l}^{H} p_{n}\right)+Q_{n k} \geq \tau_{n l} \\
(14), \mathbf{W}_{n k} \succeq \mathbf{0}, \Sigma_{n} \geq \mathbf{0}, \\
\forall k \in \mathcal{K}, \forall l \in \mathcal{L}, \forall s \in \mathcal{S}, \forall n, m \in \mathcal{N}_{c},
\end{gathered}
$$

where $\rho_{n} \geq 0$ is a slack variable and $c>0$ is the penalty parameter. Note that problem (15) and problem (17) have
the same optimal result and solution, and the added parameter. Note that problem (15) and problem (17) have
the same optimal result and solution, and the added terms are required to ensure the convergence of ADMM algorithm.

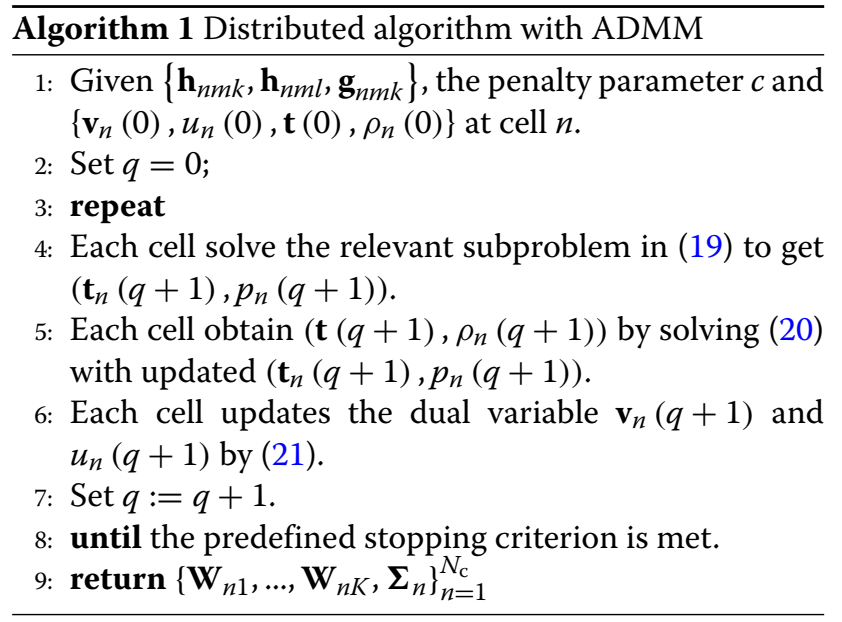

Since constraints (14), (15b), (15c), and (15d) are all convex w.r.t. the variables relevant to $n$ of problem (15), the feasible set of problem (15) can be decomposed into $N_{\mathrm{c}}$-disjointed convex sets as (16).

$$
\begin{aligned}
\Gamma_{n}=\{( & \left\{\mathbf{W}_{n k}\right\},\left\{\Sigma_{n}\right\},\left\{T_{n k}\right\},\left\{t_{m n k}\right\},\left\{E_{n s}\right\},\left\{e_{m n s}\right\},\left\{Q_{n l}\right\}, \\
& \left.\left.\left\{q_{m n l}\right\}, p_{n}\right) \mid(14),(15 b),(15 c),(15 d)\right\}, n \in \mathcal{N}_{\mathrm{c}}
\end{aligned}
$$

For clarification, we define two new vector variables as follows:

$$
\begin{gathered}
\mathbf{t} \triangleq\left[\begin{array}{l}
\left.\left[t_{121}, \ldots, t_{12 K}\right], \ldots,\left[q_{121}, \ldots, q_{12 K}\right],\right]^{T} \\
\ldots,\left[e_{N_{\mathrm{c}}\left(N_{\mathrm{c}}-1\right) 1}, \ldots, e_{N_{\mathrm{c}}\left(N_{\mathrm{c}}-1\right) K}\right]
\end{array}\right]^{T} \\
\mathbf{t}_{n} \triangleq\left[\begin{array}{l}
{\left[T_{n 1}, \ldots, T_{n K}\right],\left[Q_{n 1}, \ldots, Q_{n K}\right],} \\
{\left[E_{n 1}, \ldots, E_{n S}\right],} \\
{\left[t_{n 11}, \ldots, t_{n 1 K}\right], \ldots,\left[q_{n 11}, \ldots, q_{n 1 K}\right],} \\
\ldots,\left[e_{N_{\mathrm{c}}\left(N_{\mathrm{c}}-1\right) 1}, \ldots, e_{N_{c}\left(N_{c}-1\right) K}\right]
\end{array}\right]^{T},
\end{gathered}
$$

where $\mathbf{t}$ collects all interference and AN variables and $\mathbf{t}_{n}$ collects all interference and AN variables relevant to cell $n$ (i.e., the interference received from and sent to neighboring cells). With the two vector variables, it is easy to find a linear mapping matrix $\Omega_{n} \in\{0,1\}$, such that $\mathbf{t}_{n}=\Omega_{n} \mathbf{t}$.

As a result, the penalty augmented problem of (12) can be given by

$$
\begin{aligned}
& \min _{\left\{\mathbf{W}_{n k}\right\}\left\{\Sigma_{n}\right\}\left\{p_{n}\right\}} \sum_{n=1}^{N_{c}}\left(\begin{array}{c}
p_{m}+\frac{c}{2}\left\|\Omega_{n} \mathbf{t}-\mathbf{t}_{n}\right\|_{2}^{2} \\
\left.+\frac{c}{2}\left(p_{n}-\rho_{n}\right)^{2}\right\}, \mathbf{t}
\end{array}\right) \\
& \text { s.t. }\left(\left\{\mathbf{W}_{n k}\right\}_{k},\left\{\Sigma_{n}\right\}, \mathbf{t}_{n}, p_{n}\right) \in \Gamma_{n}, \\
& \mathbf{t}_{n}=\Omega_{n} \mathbf{t}, p_{n}=\rho_{n}, \forall n \in \mathcal{N}_{c},
\end{aligned}
$$


The second ingredient of the proposed distributed design is dual decomposition so that each transmitter can generate its own beamforming vectors and AN covariance matrix with its local CSI. The dual problem of (17) can be given by

$$
\begin{aligned}
& \max _{\substack{\mathbf{v}_{n} \in R^{N_{\mathrm{c}}(K+S)} \\
u_{n} \in R}} \min \sum_{n=1}^{N_{\mathrm{c}}}\left(\begin{array}{l}
p_{m}+\frac{c}{2}\left\|\Omega_{n} \mathbf{t}-\mathbf{t}_{n}\right\|_{2}^{2} \\
+\frac{c}{2}\left(p_{n}-\rho_{n}\right)^{2} \\
-\mathbf{v}_{n}^{T}\left(\Omega_{n} \mathbf{t}-\mathbf{t}_{n}\right) \\
-u_{n}\left(p_{n}-\rho_{n}\right)
\end{array}\right) \\
& \text { s.t. }\left(\left\{\mathbf{W}_{n k}\right\}_{k},\left\{\Sigma_{n}\right\}, \mathbf{t}_{n}, p_{n}\right) \in \Gamma_{n}, \forall n \in \mathcal{N}_{\mathrm{c}},
\end{aligned}
$$

where $\mathbf{v}_{n}$ and $u_{n}$ are associated dual variables. For given $\mathbf{v}_{n}$ and $u_{n},\left(\left\{\mathbf{W}_{m k}\right\}_{k},\left\{\boldsymbol{\Sigma}_{m}\right\}, \mathbf{t}_{m}, p_{m}\right)$ can be solved efficiently since the problem (18) is convex. So we can solve (18) by an iteration method. Particularly, at the $(q+1)$-th iteration, the problem (18) can be decomposed into $2 N_{\mathrm{c}}$ convex subproblems, i.e., $\forall n \in \mathcal{N}_{\mathrm{c}}$,

$$
\begin{aligned}
& \left\{\mathbf{t}_{n}(q+1), p_{n}(q+1)\right\} \\
& =\arg \min \left(\begin{array}{l}
p_{n}+\frac{c}{2}\left\|\Omega_{n} \mathbf{t}(q)-\mathbf{t}_{n}\right\|_{2}^{2} \\
+\frac{c}{2}\left(p_{n}-\rho_{n}(q)\right)^{2} \\
-\mathbf{v}_{n}^{T}(q) \mathbf{t}_{n}-u_{n}(q) p_{n}
\end{array}\right),
\end{aligned}
$$

and

$$
\begin{aligned}
& \left\{\mathbf{t}(q+1), \rho_{n}(q+1)\right\} \\
& =\arg \min \sum_{n=1}^{N_{\mathrm{c}}}\left(\begin{array}{l}
\frac{c}{2}\left\|\boldsymbol{\Omega}_{n} \mathbf{t}-\mathbf{t}_{n}(q+1)\right\|_{2}^{2} \\
+\mathbf{v}_{n}^{T}(q) \boldsymbol{\Omega}_{n} \mathbf{t} \\
+\frac{c}{2}\left(p_{n}(q+1)-\rho_{n}\right)^{2} \\
+u_{n}(q) \rho_{n}
\end{array}\right) .
\end{aligned}
$$

It is interesting to see that each subproblem in (19) is only relevant to the cell $n$. Thus, at the $(q+1)$-th iteration of the distributed algorithm, each cell copies with one relevant subproblem in (19) to get $\left(\mathbf{t}_{n}(q+1), p_{n}(q+1)\right)$, and then exchanges $\left(\mathbf{t}_{n}(q+1), p_{n}(q+1)\right)$ with neighboring cells so that it can obtain $\left(\mathbf{t}(q+1), \rho_{n}(q+1)\right)$ by solving (20). Then, according to ADMM, the dual variable can be updated by

$$
\left\{\begin{array}{l}
\mathbf{v}_{n}(q+1)=\mathbf{v}_{n}(q)+c\left(\boldsymbol{\Omega}_{n} \mathbf{t}(q+1)-\mathbf{t}_{n}(q+1)\right), \\
u_{n}(q+1)=u_{n}(q)+c\left(\rho_{n}(q+1)-p_{n}(q+1)\right)
\end{array}\right.
$$

For clarity, the proposed distrusted algorithm is summarized in Algorithm 1.

\section{Simulation results and discussion}

This section represents some simulation results to show the efficiency of the proposed centralized and distributed $\mathrm{AN}$-aided MCBF designs. For comparison, the non ANaided MCBF design is also simulated as a benchmark. We discuss the system performance in perspective of the SINR requirement at IRs, the allowable SINR threshold at Eves, the EH model at ERs, and the number of antennas at the transmitter. Besides, the centralized MCBF design and the distributed MCBF design are compared.

The simulation network scenario is shown in Fig. 1 where the number of cells $N_{\mathrm{c}}$ is 3 and the number of antennas $N_{\mathrm{t}}$ at each transmitter is 4 . The inter-transmitter distance $D$ is $500 \mathrm{~m}$. The number of IR, ER, and Eve in each cell is set as 2, 1 , and 1, respectively. All IRs and ERs are located about $50 \mathrm{~m}$ from their serving transmitters and all ERs are located about $10 \mathrm{~m}$ from their serving transmitters, which is close enough that the line-of-sight communication channel is expected. The channel mode adopted in our simulation is given by [29]

$$
\begin{aligned}
& \mathbf{h}_{m n k}=\beta\left(d_{m n k}\right) \cdot \psi_{m n k} \cdot \varphi \cdot \hat{\mathbf{h}}_{m n k}, \\
& \mathbf{h}_{m n l}=\beta\left(d_{m n l}\right) \cdot \psi_{m n l} \cdot \varphi \cdot \hat{\mathbf{h}}_{m n l}, \\
& \mathbf{g}_{m n s}=\beta\left(d_{m n s}\right) \cdot \psi_{m n s} \cdot \varphi \cdot \hat{\mathbf{g}}_{m n s},
\end{aligned}
$$

where $\beta(d)=10^{-\left(128.1+37.6 \log _{10}(d)\right) / 20}, d_{m n k}, d_{m n l}$, and $d_{m n s}$ denote the distance between the transmitter and the $k$-th IR, $l$-th ER, and $s$-th Eve, respectively. $\psi_{m n k}, \psi_{m n l}$, and $\psi_{m n s}$ represent the shadow fading, which follows the log-normal distribution with zero mean and standard deviation 8. $\varphi$ denotes the transmit-receive antenna gain which is set to $15 \mathrm{dBi}$, and $\hat{\mathbf{h}}_{m n k}, \hat{\mathbf{h}}_{m n l}$, and $\hat{\mathbf{g}}_{m n s}$ are the multipath fading which is modeled as Rayleigh fading for IRs and Eves and Rician fading for ERs. The noise power spectral density is $-162 \mathrm{dBm} / \mathrm{Hz}$ and the bandwidth $B$ is $10 \mathrm{MHz}$. For each IR, the SINR requirement is set as $10 \mathrm{~dB}$. For each Eve, the allowable SINR threshold is set as $-10 \mathrm{~dB}$. For the non-linear EH model of each ER, we set all $M_{l}$ as $M=24 \mathrm{~mW}$ which corresponds to the maximum harvested power at each ER. Besides, we adopt $a_{1}=\ldots=a_{L}=150$ and $b_{1}=\ldots b_{L}=0.024$. The required $\mathrm{EH}$ power $\varsigma_{s}$ at each ER is set as $10 \mathrm{~mW}$. All parameters in the simulations are as described above unless specified. In the presented simulation results, each point of the curves is averaged over 1000 channel realizations.

Figure 3 shows the required power versus the SINR requirement at each IR $\gamma_{\mathrm{u}}$. In the proposed AN-aided MCBF design, the total required power contains two parts where one is associated with beamforming vectors $\sum_{n=1}^{N_{\mathrm{c}}} \sum_{k=1}^{K}\left\|\mathbf{w}_{n k}\right\|_{2}^{2}$ and the other is associated with AN $\sum_{n=1}^{N_{\mathrm{c}}} \operatorname{Tr}\left(\Sigma_{n}\right)$. For clarity, we also plot these two parts in Fig. 3, where the power of beamforming part is marked by "Beamforming part (proposed)" and the power of AN part is marked by "AN part (proposed)." As $\gamma_{u}$ increases, more power is required in both beamforming part and AN part. Compared with the non $\mathrm{AN}$-aided MCBF, the proposed $\mathrm{AN}$-aide MCBF requires less power, which is consistent with Proposition 2.

Figure 4 compares the proposed $\mathrm{AN}$-aided MCBF under the non-linear EH model and linear EH model, where for the linear EH model, the RF-to-DC conversion efficiency 


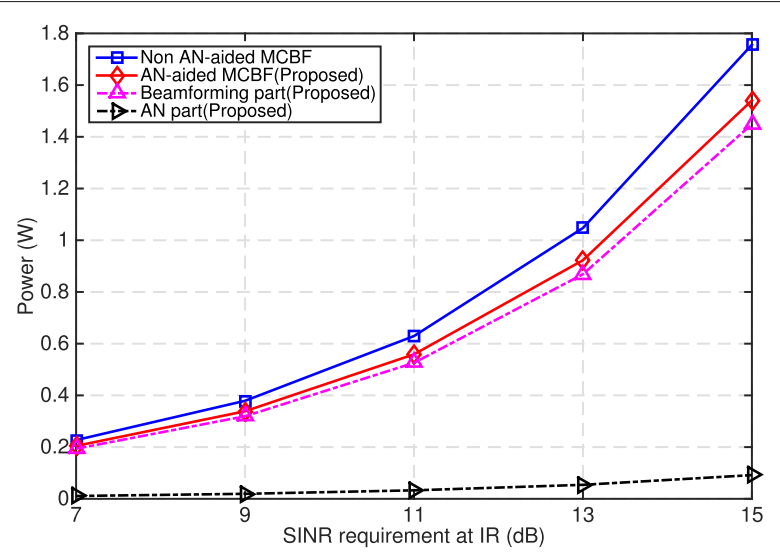

Fig. 3 Required power versus SINR requirement at each IR

$\rho$ is set as 1 and 0.5 , respectively. It is observed that for the linear EH model, the total required power increases linearly as each ER's required output DC power $\theta$ increases. For the non-linear $\mathrm{EH}$ model, the total required power also increases as $\theta$ increases, but there exists a saturation point on $\theta$ (e.g., $M=24 \mathrm{~mW}$ in our example) due to the non-linear EH circuit feature. Moreover, if the linear $\mathrm{EH}$ model with $\rho=1$ is adopted, it may result in false and deceptive output DC power at each ER. That is, when $\theta \leq M$, although less power is consumed by the linear EH model, the output DC power cannot meet the practical requirement (i.e., (9d) cannot be satisfied). When $\theta>M$, although the transmit design can still be generated by the linear EH model, (9d) also cannot be satisfied. In contrast, for the non-linear EH model, when $\theta \leq M$, (9d) can be guaranteed, and when $\theta>M$, beamforming vectors and AN cannot be generated because of the saturation limitation of practical EH circuits. Thus, the false output DC power is avoided by employing the non-linear $\mathrm{EH}$ model. Furthermore, if the linear EH model with $\rho=0.5$ is adopted, the design under the linear EH model is also feasible in practical system but more power is required

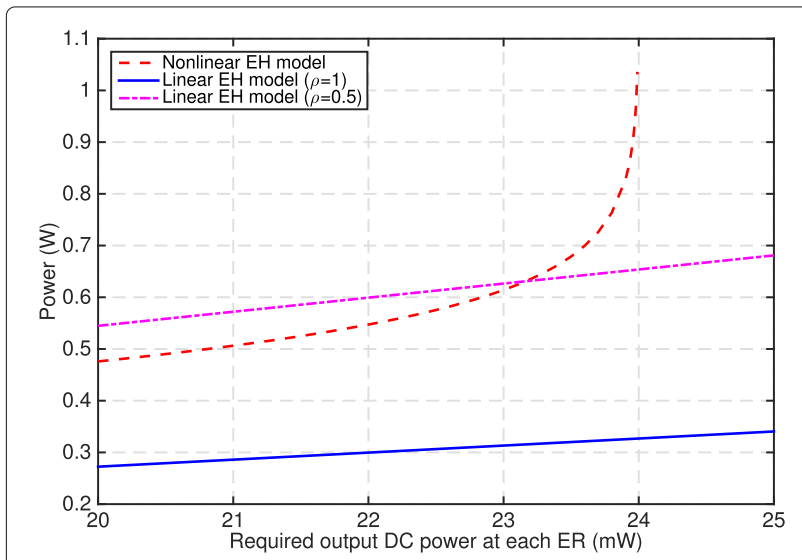

Fig. 4 Non-linear EH model versus linear EH model compared with the design under the non-linear EH model. This result demonstrates the advantage of employing the non-linear EH model.

The relation between the required power and the allowable SINR threshold at each Eve $\gamma_{e}$ is investigated in Fig. 5. Since the small $\gamma_{e}$ may make (12) infeasible, we just show the results of $\gamma_{e}$ with the range from -2 to $-10 \mathrm{~dB}$. It is observed that smaller $\gamma_{e}$ requires more total required power as well as the power in the AN part. Besides, the smaller $\gamma_{e}$, the larger gap between the AN-aided MCBF and the non $\mathrm{AN}$-aided MCBF, which indicates that to achieve high-quality secure transmission, $\mathrm{AN}$ is required.

The total required power versus $N_{\mathrm{t}}$ is shown in Fig. 6, where $N_{\mathrm{t}}$ changes from 4 to 7 . It is observed that the total required power of both the $\mathrm{AN}$-aided MCBF and the non AN-aided MCBF decreases with the increment of $N_{\mathrm{t}}$. The system performance gain between $\mathrm{AN}$-aided MCBF and non AN-aided MCBF is more obvious for relative small $N_{\mathrm{t}}$. As larger $N_{\mathrm{t}}$ indicates larger spatial DoF, it can be concluded that for system with limited spatial DoF, AN-aided MCBF is a better choice to achieve lower power consumption. Besides, it is observed that with increment of transmit antennas, the effect of AN on the total required power decreases.

Figure 7 gives the comparison of total required power between the centralized algorithm and the distributed algorithm over 20 randomly generated channel realizations, and the iteration number is 20 . It is seen that the distributed solution has a very close result to the centralized one, which means that the distributed algorithm can converge to the optimal solution to the centralized algorithm.

\section{Conclusions}

This paper studied the AN-aided MCBF for multi-cell multi-user SWIPT, where the non-linear EH model was adopted at each ER. We formulated an optimization problem to minimize the total required power by jointly

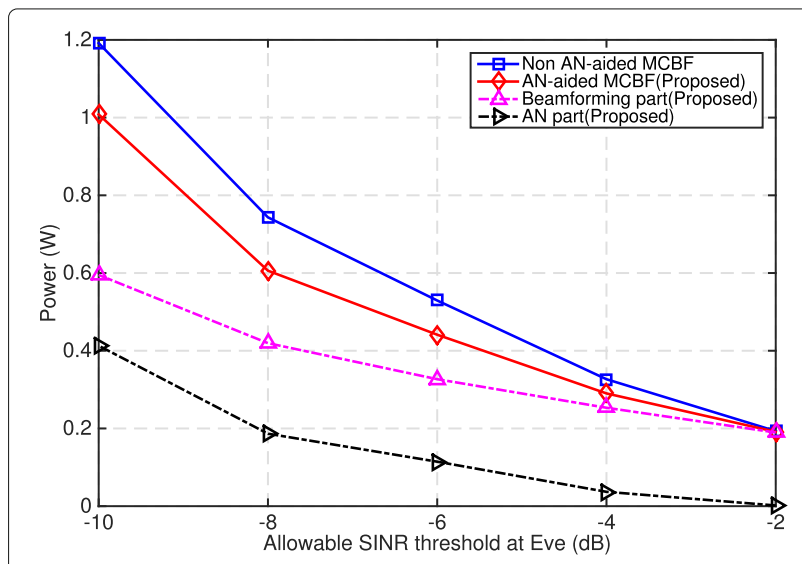

Fig. 5 Required power versus allowable SINR threshold at each Eve 


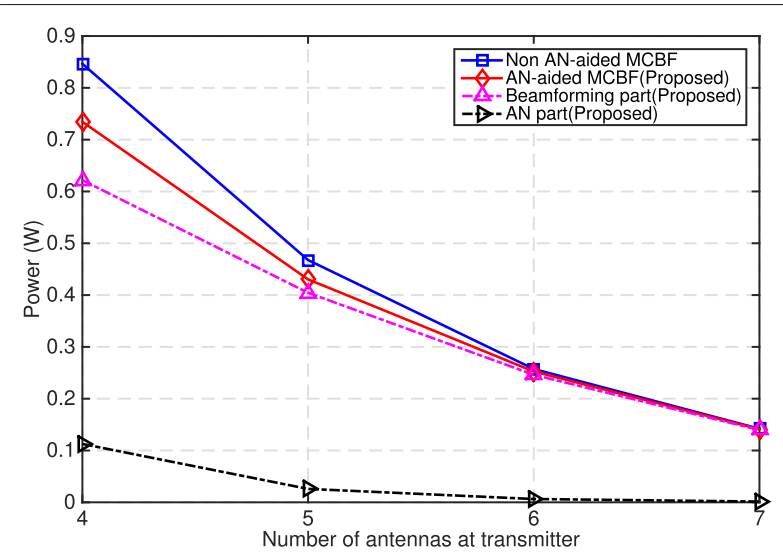

Fig. 6 Required power versus number of transmit antennas

optimizing the transmit beamforming vectors and the AN covariance matrixes at all transmitters, while guaranteeing IRs and ERs' QoS and avoiding the information intercepted by any Eve. Since the problem is non-convex, we solved it by applying SDR and proved that our proposed solution method guarantees the global optimal solution with full CSI in a centralized manner. We further presented a distributed $\mathrm{AN}$-aided MCBF design using ADMM, with which, each transmitter is able to calculate its own beamforming vectors and AN covariance matrix based on its local CSI. Simulation results showed that our proposed distributed algorithm converges to the optimal results obtained by the centralized one. Some insights are derived as follows. For the same secure transmission requirements, the proposed $\mathrm{AN}$-aided $\mathrm{MCBF}$ consumes less power than the non AN-aided MCBF. With increment of transmit antennas, the total required power decreases and the effect of $\mathrm{AN}$ on the total required power decreases. Compared with the traditional linear EH model, employing the non-linear EH model avoids false output power at the ERs and save power at the transmitter.

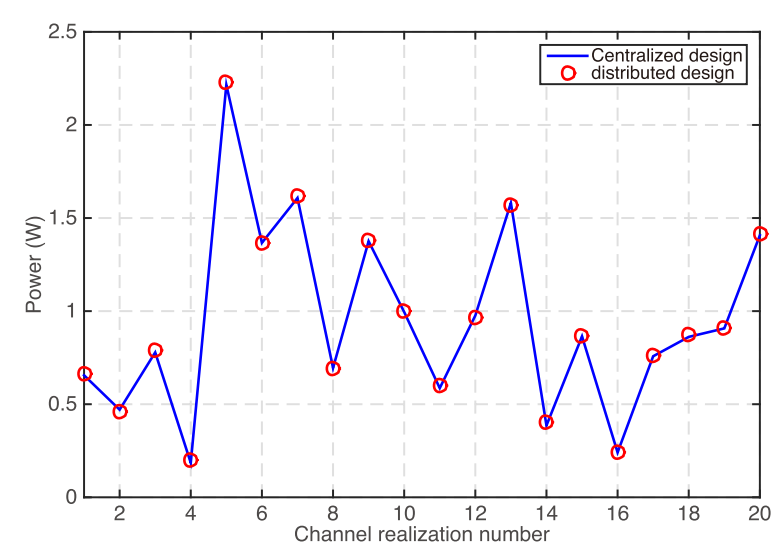

Fig. 7 Comparison of the centralized design and the distributed one

\section{Endnotes}

${ }^{1}$ The CSI of Eve can be estimated through the local oscillator power inadvertently leaked from the Eves' receiver RF frontend [32].

${ }^{2}$ Note that if there is no information leakage constraints, i.e., (9c), problem (9) could be reformulated as a convex second-order cone program (SOCP), which can be efficiently solved [29]. However, due to the information leakage constraints (9c), problem (9) cannot be solved by SOCP anymore.

\section{Appendix}

Let $\mathbf{W}_{n k}^{\star}$ represent the optimal solution to (12). $\mathbf{Y}_{n k}^{\star}, \lambda_{n k}^{\star}$, and $\mu_{n s}^{\star}$ are the corresponding optimal solutions to the dual problem of (12). The KKT equations corresponding to $\mathbf{W}_{n k}^{\star}$ in (12) can be given by

$$
\left\{\begin{array}{l}
\mathbf{I}_{N \mathrm{t}}-\mathbf{Y}_{n k}^{\star}-\boldsymbol{\Lambda}+\sum_{s=1}^{S} \frac{\mu_{n s}^{\star}}{\gamma_{\mathrm{e}}}\left(\mathbf{g}_{n n s} \mathbf{g}_{n n s}^{H}\right)=\mathbf{0}, \\
\mathbf{Y}_{n k}^{\star} \mathbf{W}_{n k}^{\star}=\mathbf{0}, \\
\mathbf{Y}_{n k}^{\star} \geq \mathbf{0}, \mu_{n s}^{\star} \geq 0, \lambda_{n k}^{\star} \geq 0, \forall k \in \mathcal{K}, \forall s \in \mathcal{S}, \forall n \in \mathcal{N}_{\mathrm{c}} .
\end{array}\right.
$$

where

$$
\boldsymbol{\Lambda}=\frac{\lambda_{n k}^{\star}}{\gamma_{u}}\left(\mathbf{h}_{n n k} \mathbf{h}_{n n k}^{H}\right)+\sum_{l=1}^{L} \frac{\lambda_{n l}^{\star}}{\tau_{n l}} \sum_{m=1}^{N} \mathbf{h}_{m n l} \mathbf{h}_{m n l}^{H},
$$

which is a PSD matrix.

So, we have rank $\left(\mathbf{Y}_{n k}^{\star} \mathbf{W}_{n k}^{\star}\right)=0$. Moreover, it is known that $\operatorname{rank}\left(\mathbf{Y}_{n k}^{\star} \mathbf{W}_{n k}^{\star}\right) \geq \operatorname{rank}\left(\mathbf{Y}_{n k}^{\star}\right)+\operatorname{rank}\left(\mathbf{W}_{n k}^{\star}\right)-N_{\mathrm{t}}$. Thus,

$$
N_{\mathrm{t}}-\operatorname{rank}\left(\mathbf{Y}_{n k}^{\star}\right) \geq \operatorname{rank}\left(\mathbf{W}_{n k}^{\star}\right) .
$$

Since $\mathbf{I}_{N \mathrm{t}}+\sum_{s=1}^{S} \frac{\mu_{n s}^{\star}}{\gamma_{\mathrm{e}}}\left(\mathbf{g}_{n n s} \mathbf{g}_{n n s}^{H}\right)$ is positive definite, we have $\mathbf{I}_{N \mathrm{t}}+\sum_{s=1}^{S} \frac{\mu_{n s}^{\star}}{\gamma_{\mathrm{e}}}\left(\mathbf{g}_{n n s} \mathbf{g}_{n n s}^{H}\right)=\mathbf{A} \mathbf{A}^{H}$ where $\mathbf{A}$ is invertible, e.g., $\operatorname{rank}(\mathbf{A})=N_{\mathrm{t}}$. Therefore, $\mathbf{Y}_{n k}^{\star}=$ $\mathbf{A}\left(\mathbf{I}_{N \mathrm{t}}-\mathbf{A}^{-1} \boldsymbol{\Lambda} \mathbf{A}^{-1}\right) \mathbf{A}$, and thus,

$$
\operatorname{rank}\left(\mathbf{Y}_{n k}^{\star}\right)=\operatorname{rank}\left(\mathbf{I}_{N \mathrm{t}}-\mathbf{A}^{-1} \boldsymbol{\Lambda} \mathbf{A}^{-1}\right) .
$$

Since $\mathbf{h}_{n n k}, \mathbf{h}_{n n l} \neq \mathbf{0}$, we have

$$
\operatorname{rank}\left(\mathbf{I}_{N \mathrm{t}}-\mathbf{A}^{-1} \boldsymbol{\Lambda} \mathbf{A}^{-1}\right)=N_{\mathrm{t}}-1,
$$

i.e., $\operatorname{rank}\left(\mathbf{Y}_{n k}^{\star}\right)=N_{\mathrm{t}}-1$. Moreover, with $\Sigma_{m} \succeq \mathbf{0}$ and $\sigma_{n s}^{2}>0$ and (12), we have $\mathbf{W}_{n k}^{\star} \neq 0$.

Following (22), if $\operatorname{rank}\left(\mathbf{Y}_{n k}^{\star}\right)=N_{\mathrm{t}}-1, \operatorname{rank}\left(\mathbf{W}_{n k}^{\star}\right) \neq 0$, $\operatorname{or} \operatorname{rank}\left(\mathbf{W}_{n k}^{\star}\right)=1$. Since rank $\left(\mathbf{W}_{n k}^{\star}\right) \neq 0, \operatorname{rank}\left(\mathbf{W}_{n k}^{\star}\right)=1$ always holds. As a result, $\operatorname{rank}\left(\mathbf{W}_{n k}^{\star}\right)=1$.

\section{Acknowledgements}

This work was supported in part by the Key Program of the National Natural Science Foundation of China (NSFC) under Grant U1334202, in part by the General Program of the NSFC under Grant 61671051, in part by the NFSC Outstanding Youth under Grant 61725101, in part by the Beijing Natural Science Foundation under Grant 4162049, and in part by the Fundamental Research Funds for the Central Universities Beijing Jiaotong University under Grants 2016JBM015 and 2017YJS063. 


\section{Authors' contributions}

Dr. YL and Dr. KX have equal contributions to the system modeling, algorithm design, performance analysis, and simulations. Dr. JL help to perform the experiments. Prof. PF and Prof. ZZ help to revise the manuscript. All authors read and approved the manuscript.

\section{Competing interests}

The authors declare that they have no competing interests.

\section{Publisher's Note}

Springer Nature remains neutral with regard to jurisdictional claims in published maps and institutional affiliations.

\section{Author details}

${ }^{1}$ School of Computer and Information Technology, Beijing Jiaotong University, Haidian District, 100044 Beijing, People's Republic of China. ${ }^{2}$ Department of Electronic Engineering, Tsinghua University, Haidian District, Beijing, People's Republic of China. ${ }^{3}$ State Key Lab of Rail Traffic Control and Safety, Beijing Jiaotong University, Haidian District, 100044 Beijing, People's Republic of China.

Received: 16 June 2017 Accepted: 17 December 2017

Published online: 14 March 2018

\section{References}

1. X Lu, P Wang, D Niyato, DI Kim, Z Han, Wireless network with rf energy harvesting: a contemporary survey. IEEE Commun. Surv. Tutor. 17(2), 757-789 (2015)

2. $\mathrm{ML} \mathrm{Ku}, \mathrm{W} \mathrm{Li}, \mathrm{Y}$ Chen, $\mathrm{KJR}$ Liu, Advances in energy harvesting communications: past, present, and future challenges. IEEE Commun. Surv. Tutor. 18(2), 1384-1412 (2016)

3. $\mathrm{B} L i, \mathrm{Z}$ Fei, Z Chu, Y Zhang, Secure transmission for heterogeneous cellular networks with wireless information and power transfer. IEEE Syst. J. PP(99), 1-12 (2017)

4. KXiong, C Chen, G Qu, P Fan, KB Letaief, Group cooperation with optimal resource allocation in wireless powered communication networks. IEEE Trans. Wirel. Commun. 16(6), 3840-3853 (2017)

5. E Boshkovska, DWK Ng, N Zlatanov, R Schober, Practical non-linear energy harvesting model and resource allocation for swipt systems. IEEE Commun. Lett. 19(12), 2082-2085 (2015)

6. KXiong, B Wang, KR Liu, Rate-energy region of swipt for mimo broadcasting under nonlinear energy harvesting model. IEEE Trans. Wirel. Commun. 16(8), 5147-5161 (2017)

7. E Boshkovska, R Morsi, DWK Ng, R Schober, in Proc. IEEE ICC. Power allocation and scheduling for SWIPT systems with non-linear energy harvesting model. (Kuala Lumpur, 2016), pp. 1-6

8. K Xiong, P Fan, Y Lu, KB Letaief, Energy efficiency with proportional rate fairness in multirelay OFDM networks. IEEE J. Sel. Areas Commun. 34(5), 1431-1447 (2016)

9. D Lopez-Perez, X Chu, AV Vasilakos, H Claussen, Power minimization based resource allocation for interference mitigation in OFFMA Femtocell networks. IEEE J. Sel. Areas Commun. 32(2), 333-344 (2014)

10. KXiong, P Fan, Z Xu, HC Yang, KB Letaief, Optimal cooperative beamforming design for MIMO decode-and-forward relay channels. IEEE Trans. Signal Process. 62(6), 1476-1489 (2014)

11. KXiong, P Fan, C Zhang, K Letaief, Wireless information and energy transfer for two-hop non-regenerative MIMO-OFDM relay networks. IEEE J. Sel. Areas Commun. 33(8), 1595-1611 (2015)

12. H Dahrouj, W Yu, Coordinated beamforming for the multicell multi-antenna wireless system. IEEE Trans. Wirel. Commun. 9(5), 1748-1759 (2010)

13. Y Lu, K Xiong, PY Fan, ZD Zhong, in Proc. IEEE ICC. Optimal coordinated beamforming with artificial noise for secure transmission in multi-cell multi-user networks. (Paris, 2017), pp. 1-6

14. Y Lu, K Xiong, PY Fan, ZD Zhong, Optimal multi-cell coordinated beamforming for downlink high-speed railway communications. IEEE Trans. Veh. Technol. 66(10), 9603-9608 (2017)

15. KXiong, P Fan, HC Yang, KB Letaief, Space-time network coding with overhearing relays. IEEE Trans. Wirel. Commun. 13(7), 3567-3582 (2014)

16. S Sim, S-J Han, J-S Park, S-C Lee, in IEEE Commun. Mag., vol. 47, Seamless IP mobility support for flat architecture mobile WiMAX networks. (2009), pp. $142-148$
17. R Negi, S Goel, in Proc. IEEE VTC-Fall. Secret communication using artificial noise. (Dallas, 2005), pp. 1906-1910

18. WC Liao, TH Chang, WK Ma, CY Chi, Qos-based transmit beamforming in the presence of eavesdroppers: an optimized artificial-noise-aided approach. IEEE Trans. Signal Process. 59(3), 1202-1216 (2011)

19. W Mei, Z Chen, C Huang, in Proc. IEEE ICASSP. Robust artificial-noise aided transmit design for multi-user MISO systems with integrated services. (Shanghai, 2016), pp. 3856-3860

20. Y Tang, J Xiong, D Ma, X Zhang, Robust artificial-noise aided transmit design for MISO wiretap channels with channel uncertainty. IEEE Commun. Lett. 17(11), 2096-2099 (2013)

21. Q Li, WK Ma, AMC So, in Proc. IEEE ICASSP. Robust artificial noise-aided transmit optimization for achieving secrecy and energy harvesting. (Florence, 2014), pp. 1596-1600

22. $\mathrm{B} \mathrm{Li}, \mathrm{Z}$ Fei, $\mathrm{H}$ Chen, Robust artificial noise-aided secure beamforming in wireless-powered non-regenerative relay networks. IEEE Access. 4 , 7921-7929 (2016)

23. WD Mei, B Fu, LX Li, Z Chen, C Huang, in Proc. IEEE ICC. Artificial-noise aided transmit design for multi-user MISO systems with service integration and energy harvesting, (Kuala Lumpur, 2016), pp. 219-225

24. M Zhang, Y Liu, R Zhang, Artificial-noise aided secrecy information and power transfer in OFDMA systems. IEEE Trans. Wirel. Commun. 15(4), 3085-3096 (2016)

25. DWK Ng, ES Lo, R Schober, Robust beamforming for secure communication in systems with wireless information and power transfer. IEEE Trans. Wirel. Commun. 13(8), 4599-4615 (2014)

26. A Salem, KA Hamdi, KM Rabie, Physical layer security with RF energy harvesting in AF multi-antenna relaying networks. IEEE Trans. Commun. 64(7), 3025-3038 (2016)

27. M Tian, X Huang, Q Zhang, J Qin, Robust an-aided secure transmission scheme in MISO channels with simultaneous wireless information and power transfer. IEEE Signal Process. Lett. 22(6), 723-727 (2015)

28. C Shen, TH Chang, KY Wang, Z Qiu, CY Chi, Distributed robust multi-cell coordinated beamforming with imperfect CSI: an ADMM approach. IEEE Trans. Signal Process. 60(6), 2988-3003 (2012)

29. H Dahrouj, W Yu, Coordinated beamforming for the multicell multi-antenna wireless system. IEEE Trans. Wirel. Commun. 9(5), 1748-1759 (2010)

30. M Li, LB Collings, SV Hanl, C Liu, P Whiting, Multicell coordinated scheduling with multiuser zero-forcing beamforming. IEEE Trans. Wirel. Commun. 15(2), 827-842 (2016)

31. S Lakshminarayana, M Assaad, M Debbah, Coordinated multicell beamforming for massive MIMO: a random matrix approach. IEEE Trans. Inf. Theory. 61 (6), 3387-3412 (2015)

32. A Mukherjee, AL Swindlehurst, in Proc. IEEE ICASSP. Detecting Passive Eavesdroppers in the MIMO Wiretap Channel. (2012), pp. 2809-2812

\section{Submit your manuscript to a SpringerOpen ${ }^{\circ}$ journal and benefit from:}

- Convenient online submission

- Rigorous peer review

- Open access: articles freely available online

- High visibility within the field

- Retaining the copyright to your article

Submit your next manuscript at $\boldsymbol{~ s p r i n g e r o p e n . c o m ~}$ 